\title{
Simulation of rainfall time series from different climatic regions using the direct sampling technique
}

\author{
F. Oriani ${ }^{1}$, J. Straubhaar ${ }^{1}$, P. Renard ${ }^{1}$, and G. Mariethoz ${ }^{2}$ \\ ${ }^{1}$ Centre for Hydrogeology and Geothermics, University of Neuchâtel, Neuchâtel, Switzerland \\ ${ }^{2}$ School of Civil and Environmental Engineering, University of New South Wales, Sydney, New South Wales, Australia \\ Correspondence to: F. Oriani (fabio.oriani@unine.ch)
}

Received: 21 February 2014 - Published in Hydrol. Earth Syst. Sci. Discuss.: 21 March 2014

Revised: 30 June 2014 - Accepted: 2 July 2014 - Published: 14 August 2014

\begin{abstract}
The direct sampling technique, belonging to the family of multiple-point statistics, is proposed as a nonparametric alternative to the classical autoregressive and Markovchain-based models for daily rainfall time-series simulation. The algorithm makes use of the patterns contained inside the training image (the past rainfall record) to reproduce the complexity of the signal without inferring its prior statistical model: the time series is simulated by sampling the training data set where a sufficiently similar neighborhood exists. The advantage of this approach is the capability of simulating complex statistical relations by respecting the similarity of the patterns at different scales. The technique is applied to daily rainfall records from different climate settings, using a standard setup and without performing any optimization of the parameters. The results show that the overall statistics as well as the dry/wet spells patterns are simulated accurately. Also the extremes at the higher temporal scale are reproduced adequately, reducing the well known problem of overdispersion.
\end{abstract}

\section{Introduction}

The stochastic generation of rainfall time series is a key topic for hydrological and climate science applications: the challenge is to simulate a synthetic signal honoring the high-order statistics observed in the historical record, respecting the seasonality and persistence from the daily to the higher temporal scales. Among the different proposed techniques, exhaustively reviewed by Sharma and Mehrotra (2010), the most commonly adopted approach to the problem since the 1960s is the Markov-chain (MC) simulation: in its classical form, it is a linear model which cannot simulate the variability and persistence at different scales. Solutions to deal with this limitation consist of introducing exogenous climatic variables and large-scale circulation indexes (Hay et al., 1991; Bardossy and Plate, 1992; Katz and Parlange, 1993; Woolhiser et al., 1993; Hughes and Guttorp, 1994; Wallis and Griffiths, 1997; Wilby, 1998; Kiely et al., 1998; Hughes et al., 1999), lower-frequency daily rainfall covariates (Wilks, 1989; Briggs and Wilks, 1996; Jones and Thornton, 1997; Katz and Zheng, 1999) or an index based on the short-term daily historical or previously generated record (Harrold et al., 2003a, b; Mehrotra and Sharma, 2007a; Mehrotra and Sharma, 2007b) as conditioning variables for the estimation of the MC parameters. By doing this, nonlinearity is introduced in the prior model, and the MC parameters change with time as a function of some specific lowfrequency fluctuations. An alternative method proposed is model nesting (Wang and Nathan, 2002; Srikanthan, 2004, 2005; Srikanthan and Pegram, 2009), which implies the correction of the generated daily rainfall using a multiplicative factor to compensate the bias in the higher-scale statistics. These techniques generally allow a better reproduction of the statistics up to the annual scale, but they imply the estimation of a more complex prior model and cannot completely capture a complex dependence structure.

In this paper, we propose the use of some lower-frequency covariates of daily rainfall in a completely unusual framework: the direct sampling (DS) technique (Mariethoz et al., 2010), which belongs to multiple-point statistics (MPS). Introduced by Guardiano and Srivastava (1993) and widely 
developed during the last decade (Strebelle, 2002; Allard et al., 2006; Zhang et al., 2006; Arpat and Caers, 2007; Honarkhah and Caers, 2010; Straubhaar et al., 2011; Tahmasebi et al., 2012), MPS is a family of geostatistical techniques widely used in spatial-data simulations and particularly suited to pattern reproduction. MPS algorithms use a training image; i.e., a data set to evaluate the probability distribution (pdf) of the variable simulated at each point (in time or space), conditionally to the values present in its neighborhood. In the particular case of the DS technique, the concept of training image is taken to the limit by avoiding the computation of the conditional pdf and making a random sampling of the historical data set where a pattern similar to the conditioning data is found. If the training data set is representative enough, these techniques can easily reproduce high-order statistics of complex natural processes at different scales. MPS has already been successfully applied to the simulation of spatial rainfall occurrence patterns (Wojcik et al., 2009). In this paper, we test the DS technique on the simulation of daily rainfall time series. The aim is to reproduce the complexity of the rainfall signal up to the decennial scale, simulating the occurrence and the amount at the same time with the aid of a multivariate data set. Similar algorithms performing a multivariate simulation had been previously developed by Young (1994) and Rajagopalan and Lall (1999) using a bootstrap-based approach. As discussed in detail in Sect. 2.3, the advantage of DS with respect to the mentioned techniques is the possibility to have a variable high-order time-dependence, without incurring excessive computation since the estimation of the $n$-dimensional conditional pdf is not needed. Moreover, we propose a standard setup for rainfall simulation: an ensemble of auxiliary variables and fixed values for the main parameters required by the direct sampling algorithm, suitable for the simulation of any stationary rainfall time series, without the need of calibration. The technique is tested on three time series from different climatic regions of Australia. The paper is organized as follows: in Sect. 2 the DS algorithm is introduced and compared with the existing resampling techniques. The data set used, the proposed setup and the method of evaluation are described in Sect. 3. The statistical analysis of the simulated time series is presented and discussed in Sect. 4 and Sect. 5 is dedicated to the conclusions.

\section{Methodology}

In this section we recall the basics of multiple-point statistics and we focus on the direct sampling algorithm. The data set used is then presented as well as the methods of evaluation.

\subsection{Background on multiple-point statistics}

Before entering in the details of the DS algorithm, let us introduce some common elements of MPS. The whole information used by MPS to simulate a certain process is based on the training image (TI) or training data set: the data set constituted of one or more variables used to infer the statistical relations and occurrence probability of any datum in the simulation. The TI may be constituted of a conceptual model instead of real data, but in the case of the rainfall time series it is more likely to be a historical record of rainfall measurements. The simulation grid (SG) is a time-referenced vector in which the generated values are stored during the simulation. Following a simulation path which is usually random, the SG is progressively filled with simulated values and becomes the actual output of the simulation. The conditioning data are a group of given data (e.g., rainfall measurements) situated in the SG. Being already informed, no simulation occurs at those time steps. The presence of conditioning data affects, in their neighborhood, the conditional law used for the simulation and limits the range of possible patterns. MPS, as well some MC-based algorithms for rainfall simulation (see Sect. 1), may include the use of auxiliary variables to condition the simulation of the target variable. Auxiliary variables may either be known (fully or partially) and used to guide the simulation, or they may be unknown but still cosimulated because their structures contain important characteristics of the signal. For rainfall time series it could be, for example, covariates of the original or previously simulated data (e.g., the number of wet days in a past period), a correlated variable for which the record is known, a theoretical variable that imposes a periodicity or a trend (e.g., a sinusoid function describing the annual seasonality over the data). Finally, the search neighborhood is a moving window - i.e., the portion of time series located in the past and future neighborhood of each simulated value - used to retrieve the data event; i.e., the group of time-referenced values used to condition the simulation.

\subsection{The direct sampling algorithm}

Classical MPS implementations create a catalog of the possible neighbor patterns to evaluate the conditional probability of occurrence for each event with respect to the considered neighborhood. This may imply a significant amount of memory and always limits the application to categorical variables. On the contrary, the DS algorithm generates each value by sampling the data from the TI where a sufficiently similar neighborhood exists. The DS implementation used in this paper is called DeeSse (Straubhaar, 2011). The following is the main workflow of the algorithm for the simulation of a single variable. For the multivariate case see the last paragraph of this section.

Let us denote $\boldsymbol{x}=\left[x_{1}, \ldots, x_{n}\right]$ the time vector representing the SG, $\boldsymbol{y}=\left[y_{1}, \ldots, y_{m}\right]$ the one representing the TI and $Z(\cdot)$ the target variable, object of the simulation, defined at each element of $\boldsymbol{x}$ and $\boldsymbol{y}$. Before the simulation begins, all continuous variables are normalized using the transformation $Z \longmapsto Z \cdot(\max (Z)-\min (Z))^{-1}$ in order to have distances 
(see step 3 ) in the range [0, 1]. During the simulation, the uninformed time steps of the SG are visited in a random order. The random simulation path $t \in\{1,2, \ldots, M\}$ is obtained by sampling without replacement the discrete uniform distribution $U(1, M)$ where $M$ is the SG length. At each uninformed $x_{t}$, the following steps are executed:

1. The data event $\boldsymbol{d}\left(x_{t}\right)=\left\{Z\left(x_{t+h_{1}}\right), \ldots, Z\left(x_{t+h_{n}}\right)\right\}$ is retrieved from the SG according to a fixed neighborhood of radius $R$ centered on $x_{t}$. It consists of at most $N$ informed time steps, closest to $x_{t}$. This defines a set of lags $H=\left\{h_{1}, \ldots, h_{n}\right\}$, with $\left|h_{i}\right| \leq R$ and $n \leq N$. The size of $\boldsymbol{d}\left(x_{t}\right)$ is therefore limited by the user-defined parameter $N$ and the available informed time steps inside the search neighborhood.

2. A random time step $y_{i}$ in $\boldsymbol{y}$ is visited and the corresponding data event $\boldsymbol{d}\left(y_{i}\right)$, defined according to $H$, is retrieved to be compared with $\boldsymbol{d}\left(x_{t}\right)$.

3. A distance $D\left(\boldsymbol{d}\left(x_{t}\right), \boldsymbol{d}\left(y_{i}\right)\right)$ - i.e., a measure of dissimilarity between the two data events - is calculated. For categorical variables (e.g., the dry/wet rainfall sequence), it is given by the formula

$$
\begin{aligned}
D\left(\boldsymbol{d}\left(x_{t}\right), \boldsymbol{d}\left(y_{i}\right)\right) & =\frac{1}{n} \sum_{j=1}^{n} a_{j}, \\
a_{j} & =\left\{\begin{array}{lll}
1 & \text { if } & Z\left(x_{j}\right) \neq Z\left(y_{j}\right) \\
0 & \text { if } & Z\left(x_{j}\right)=Z\left(y_{j}\right),
\end{array}\right.
\end{aligned}
$$

while for continuous variables the following one is used:

$D\left(\boldsymbol{d}\left(x_{t}\right), \boldsymbol{d}\left(y_{i}\right)\right)=\frac{1}{n} \sum_{j=1}^{n}\left|Z\left(x_{j}\right)-Z\left(y_{j}\right)\right|$,

where $n$ is the number of elements of the data event. The elements of $\boldsymbol{d}\left(x_{t}\right)$, independently from their position, play an equivalent role in conditioning the simulation of $Z\left(x_{t}\right)$. Note that, using the above distance formulas, the normalization is not needed for categorical variables, while for the continuous ones it ensures distances in the range $[0,1]$.

4. If $D\left(\boldsymbol{d}\left(x_{t}\right), \boldsymbol{d}\left(y_{i}\right)\right)$ is below a fixed threshold $T-$ i.e., the two data events are sufficiently similar - the iteration stops and the datum $Z\left(y_{i}\right)$ is assigned to $Z\left(x_{t}\right)$. Otherwise, the process is repeated from point 2 until a suitable candidate $\boldsymbol{d}\left(y_{i}\right)$ is found or the prescribed TI fraction limit $F$ is scanned.

5. If a TI fraction $F$ has been scanned and the distance $D\left(\boldsymbol{d}\left(x_{t}\right), \boldsymbol{d}\left(y_{i}\right)\right)$ is above $T$ for each visited $y_{i}$, the datum $Z\left(y_{i}^{*}\right)$ minimizing this distance is assigned to $Z\left(x_{t}\right)$.
This procedure is repeated for the simulation at each $x_{t}$ until the entire SG is covered. Figure 1 illustrates the iterative simulation using the DS technique and stresses some of its peculiarities. First, simulating $Z\left(x_{t}\right)$ in a random order allows $\boldsymbol{x}$ to be progressively populated at nonconsecutive time steps. Therefore, the simulation at each $x_{t}$ can be conditioned on both past and future, as opposed to the classical Markovchain techniques, that use a linear simulation path starting from the beginning of the series, allowing conditioning on past only.

In the early iterations, the closest informed time steps used to condition the simulation are located far from $x_{t}$ and its number is limited by the search window; i.e., conditioning is mainly based on large past and future time lags. On the contrary, the final iterations dispose of a more populated SG, conditioning is thus done on small time lags since only the closest $N$ values are considered. This variable time-lag principle may not respect the autocorrelation on a specific time lag rigorously, but it should preserve a more complex statistical relationship, which cannot be explored exhaustively using a fixed-dependence model.

The DS can simulate multiple variables together similarly to the univariate case, dealing with a vector of variables $\boldsymbol{Z}\left(x_{t}\right)$ and considering a data event $\boldsymbol{d}_{k}$ different for each $k$ th variable, defined by $N_{k}$ and $R_{k}$. Unlike the implementation presented in Mariethoz et al. (2010), DeeSse also uses a specific acceptance threshold $T_{k}$ for each variable. Step 3 of the algorithm is repeated until a candidate with a distance below the threshold for all variables is found. If this condition is not met, the scan stops at the prescribed TI fraction $F$ and the error for each candidate $y_{i}$ and $k$ th variable is computed with the following formula: $E_{k}\left(y_{i}\right)=\left(D\left(\boldsymbol{d}_{k}\left(x_{t}\right)\right.\right.$, $\left.\left.\boldsymbol{d}_{k}\left(y_{i}\right)\right)-T_{k}\right) T_{k}^{-1}$, where $D(\cdot, \cdot)$ is defined as in step 3. Finally, the candidate minimizing $\max \left(\boldsymbol{E}\left(y_{i}\right)\right)$ is assigned to $\boldsymbol{Z}\left(x_{t}\right)$. Note that the entire data vector $\boldsymbol{Z}\left(x_{t}\right)$ is simulated in one iteration, reproducing exactly the same combination of values found for all the variables at the sampled time step, excluding the conditioning data, already present in the SG. This feature, although reducing the variability in the simulation, has been adopted to accurately reproduce the correlation between variables.

\subsection{Comparison with existing resampling techniques}

The resampling principle is at the base of some already proposed techniques for rainfall and hydrologic time-series simulation. There exist two principal families of resampling techniques: the block bootstrap (Vogel and Shallcross, 1996; Srinivas and Srinivasan, 2005; Ndiritu, 2011), which implies the resampling with replacement of entire pieces of time series with the aim of preserving the statistical dependence at a scale minor than the block size, and the $k$-nearest neighbor bootstrap (k-NN), based on single-value resampling using a pattern similarity rule. This latter family of techniques, introduced by Efron (1979) and inspired by the jackknife 


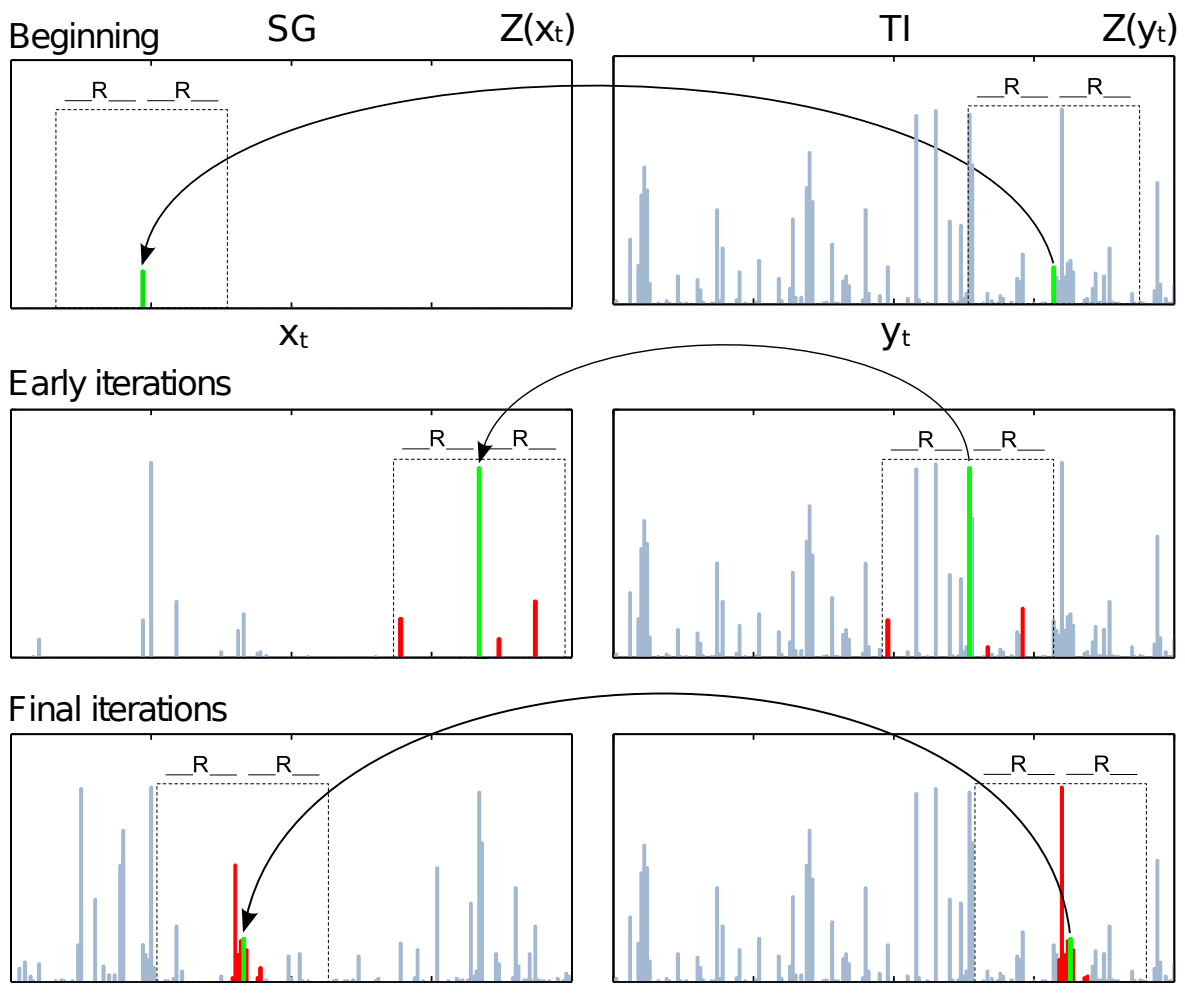

Figure 1. Sketch of the sequential simulation of a rainfall time series performed by the direct sampling technique: the dashed rectangle represents the search neighborhood of radius $R$, the datum being simulated is in green and the ones composing the data event are in red. Note the nonexact match between the data event in the SG and the one in the TI.

variance estimation, has seen several developments in hydrology (Young, 1994; Lall and Sharma, 1996; Lall et al., 1996; Rajagopalan and Lall, 1999; Buishand and Brandsma, 2001; Wojcik and Buishand, 2003; Clark et al., 2004). Having different points in common with the DS technique, its general framework is briefly presented in the following. Each datum inside the historical record is characterized by a vector $\boldsymbol{d}_{t}$ of predictor variables, analogous to the data event for DS. For example, to generate $Z\left(x_{t}\right)$ one could use $\boldsymbol{d}_{t}=\left[Z\left(x_{t-1}\right)\right.$, $Z\left(x_{t-2}, U\left(x_{t}\right), U\left(x_{t-1}\right)\right]$, meaning that the simulation is conditioned to the two previous time steps of $Z$ and the present and previous time steps of $U$, a correlated variable. In the predictor variables space $\mathbb{D}$, the historical data as well as $Z\left(x_{t}\right)$, which still has to be generated, are represented as points whose coordinates are defined by $\boldsymbol{d}_{t}$. Consequently, proximity in $\mathbb{D}$ corresponds to similarity of the conditioning patterns. $Z\left(x_{t}\right)$ is simulated by sampling an empirical pdf constructed on the $k$ points closest to $Z(x t)$; the closer the point is, the higher is the probability to sample the corresponding historical datum. Proposed variations of the algorithm include transformations of the predictor variables space, the application of kernel smoothing to the k-NN pdf to increase the variability beyond the historical values, and different methods to estimate the parameters of the model; e.g., $k$ and the kernel bandwidth.
Going back to DS, the similarities with the k-NN bootstrap are that both (i) make a resampling of the historical record conditioned by an ensemble of auxiliary/predictor variables, and (ii) compute a distance as a measure of dissimilarity between the simulating time step and the candidates considered for resampling. Nevertheless, there are several points of divergence in the rationale of the techniques: (i) in the $\mathrm{k}-\mathrm{NN}$ bootstrap, the distance is used to evaluate the resampling probability, while in the DS it is used to evaluate the resampling possibility. This means that, using the k-NN resampling, the conditional pdf is a function of the distance, while in the DS the distance is only used to define its support. In fact, using the DS, the space $\mathbb{D}$ is not restricted to the $k$ nearest neighbors but it is bounded by the distance thresholds: outside the boundary, the resampling probability is zero, while inside, it follows the occurrence of the data in the scanned TI fraction, without being a function of the pattern resemblance. Only in cases where no candidate is found, it is the closest neighbor outside the bounded portion of $\mathbb{D}$ to be chosen for resampling. The latter can be considered as an exceptional condition which usually does not lead to a good simulation and seldom occurs using an appropriate setup and training data set. (ii) Using the DS, the conditional pdf remains implicit, its computation is not needed; i.e., the historical record is randomly visited instead and the 
first datum presenting a distance below the threshold is sampled. This is an advantage since it avoids the problem of the high-dimensional conditional pdf estimation which limits the degree of conditioning in bootstrap techniques (Sharma and Mehrotra, 2010). (iii) The k-NN technique considers a fixed time-dependence, while it varies during the simulation in the case of DS. (iv) Finally, the simulation path (in the SG) is always linear in the k-NN technique, while it is random using DS, allowing conditioning on future time steps of the target variable.

\section{Application}

The data set chosen for this study is composed of three daily rainfall time series from different climatic regions of Australia: Alice Springs (hot desert), with a very dry rainfall regime and long droughts, Sydney (temperate), with a far wetter climate due to its proximity to the ocean, and Darwin (tropical savannah), showing an extreme variability between the dry and wet seasons.

Table 1 presents the data set used: the chosen stations provide a considerable record of about 70 years for Darwin and Alice Springs and 150 years for Sydney. Any gaps or trends have been explicitly kept to test the behavior of the algorithm with incomplete or nonstationary data sets. The direct sampling algorithm treats gaps in the time series in a simple way: each data event found in the TI is rejected if it contains any missing data. This allows incomplete training images to be dealt with in a safe way, but, as one could expect, a large quantity of missing data, especially if sparsely distributed, may lead to a poor simulation. Mariethoz and Renard (2010) show how DS can be used for data reconstruction.

Since rainfall is a complex signal exhibiting not only multiscale time-dependence but also intermittence, the classical approach is to split the daily time-series generation in two steps: the occurrence model, where the dry/wet daily sequence is generated using a Markov chain, and the amount model, where the rainfall amount is simulated on wet days using an estimation of the conditional pdf (e.g., Coe and Stern, 1982). The simulation framework proposed here is radically different: we use the direct sampling technique to generate the complete time series in one step, simulating multiple variables together. In particular, the TI used is based on the past daily rainfall record and composed of the following variables (Table 2): (1) the average rainfall amount on a 365-day centered moving window (365 MA; mm), (2) the moving sum of the current and the previous day amounts (2 MS; mm), (3) and (4) two out-of-phase triangular functions ( $\operatorname{tr} 1$ and $\operatorname{tr} 2)$ with frequency of 365.25 days, similar to trigonometric coordinates expressing the position of the day in the annual cycle, (5) the dry/wet sequence (i.e., a categorical variable indicating the position of a day inside the rainfall pattern: 1 - wet, 0 - dry, 2 - solitary wet, and 3 - wet day at the beginning or at the end of a wet spell), and (6) the daily
Table 1. Summary of the data set used.

\begin{tabular}{llccc}
\hline Location & Station & $\begin{array}{c}\text { Period } \\
\text { (years) }\end{array}$ & $\begin{array}{c}\text { Record } \\
\text { length } \\
\text { (days) }\end{array}$ & $\begin{array}{c}\text { Missing } \\
\text { data } \\
\text { (days) }\end{array}$ \\
\hline Alice Springs & A. S. Airport & $1940-2013$ & 26347 & 305 \\
Sydney & S. Observatory Hill & $1858-2013$ & 56662 & 184 \\
Darwin & D. Airport & $1941-2013$ & 26356 & 0 \\
\hline
\end{tabular}

rainfall amount, which is the target of the simulation. The first two auxiliary variables are covariates used to force the algorithm to preserve the interannual structure and the dayto-day correlation, which are known to exist a priori. The others are used to reproduce the dry/wet pattern and the annual seasonality accurately. Moreover, any unknown dependence in the daily rainfall signal is generically taken into account in the simulation by using a data event of variable length as explained in Sect. 2.2. It has to be remarked that, apart from (3) and (4), which are known deterministic functions imposed as conditioning data, the rest of the auxiliary variables are transformations of the rainfall datum, automatically computed on the TI and cosimulated with the daily rainfall.

To summarize, the main parameters of the algorithm are the following: the maximum scanned TI fraction $F \in(0,1]$, the search neighborhood radius $R$, the maximum number of neighbors $N$, both expressed in number of elements of the time vector, and the distance threshold $T \in(0,1]$. Recall that, apart from $F$, each parameter is set independently for each simulated variable. The setup shown in Table 2 is used together with $F=0.5$ and proposed as a standard for daily rainfall time series. A sensitivity analysis, not shown here, confirmed the generality of this setup which is not the result of a numerical optimization on a specific data set, but it is rather in accordance to the criteria used to define the order and extension of the variable time-dependence, as shown below. Applying it to any type of single-station daily rainfall data set, the user should obtain a reliable simulation without needing to change any parameter or give supplementary information. An additional refinement of the setup is also possible, keeping in mind the following general rules:

- $R$ limits the maximum time-lag dependence in the simulation and should be set according to the length of the largest sufficiently repeated structure or frequency in the signal that has to be reproduced. Being interested to condition the simulation upon the inter-annual fluctuations (visible in the 10-year MA time series in Fig. 9), we set $R_{365 \mathrm{MS}}=R_{\text {rainfall }}=5000$ for the $365 \mathrm{MS}$ and daily rainfall variables. We recommend keeping $R$ below the half of the training data set's total length, to condition upon sufficiently repeated structures only. Regarding dry/wet pattern conditioning, we prefer limiting the variable time-dependence within a 21-day window $\left(R_{\mathrm{dw}}=10\right)$. This window should be set between the median and the maximum of the wet-spell-length 
Table 2. Standard setup proposed for rainfall simulation. The parameters are search window radius $R$, maximum number of neighbors $N$ and distance threshold $T$. The variables are (1) the 365-day moving average (365 MA), (2) the moving sum of the current and the previous day amounts (2 MS), (3) and (4) annual seasonality triangular functions ( $\operatorname{tr} 1$ and tr2), (5) the dry/wet sequence (dw), and (6) the daily rainfall amount as the target variable. On the right, a portion of multivariate TI is given as example.

\begin{tabular}{|c|c|c|c|c|}
\hline Variable & $R$ & $N$ & $T$ & \\
\hline (1) $365 \mathrm{MA}$ & 5000 & 21 & 0.05 & \\
\hline (2) $2 \mathrm{MS}$ & 1 & 1 & 0.05 & \\
\hline (3) $\operatorname{tr} 1$ & 1 & 1 & 0.05 & \\
\hline (4) $\operatorname{tr} 2$ & 1 & 1 & 0.05 & \\
\hline (5) $\mathrm{dw}$ & 10 & 5 & 0.05 & 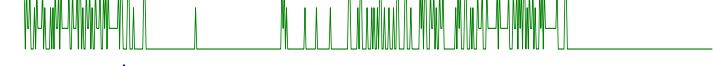 \\
\hline (6) rainfall & 5000 & 21 & 0.05 & I000 IdaysI \\
\hline
\end{tabular}

distribution, in order to properly catch the continuity of the rainfall events over multiple days.

- $N$ controls the complexity of the conditioning structure but also influences the specific time-lag dependence. For instance, if one increases $N$, higher-order dependencies are represented, but the weight accorded to a specific neighbor in evaluating the distance between patterns becomes lower. This leads to a less-accurate specific-time-lag conditioning, but a more complex time-dependence is respected on average. For the rainfall amount and $365 \mathrm{MA}$ variables, $N \ll R$ follows the same setup rule as for $R_{\mathrm{dw}}$. In this way, in the initial iterations, the conditioning neighbors will be sparse in a 10001-day window $(R=5000)$ to respect lowfrequency fluctuations, whereas, in the final iterations, they will be contained in a $N$-day window to respect the within-spell variability. The standard value proposed here $\left(N_{365 \mathrm{MA}}=N_{365 \mathrm{MA}}=21\right)$ corresponds approximately to the spell-distribution median of the Darwin time series, remaining in the appropriate range for the other considered climates. Conversely, $N_{\mathrm{dw}}$ is kept lower in order to focus the conditioning on the smallscale dry/wet pattern. $N_{\mathrm{dw}}=5$ gave in general the best result in terms of dry/wet pattern reproduction.

- For $2 \mathrm{MS}$, tr 1 and tr2, the time-dependence is limited to lag 1 by using $N=R=1$. This combination should not be changed since we have no interest in expanding or varying the time-lag dependence for the mentioned variables.

- $T$ determines the tolerance in accepting a pattern. The sensitivity analysis done until now on different types of heterogeneities (Meerschman et al., 2013) confirmed that the optimum generally lies in the interval $[0.01,0.07]$ (1-7\% of the total variation). Higher $T$ values usually lead to poorly simulated patterns, but lower ones may induce a bias in the marginal distribution and increase the phenomenon of verbatim copy; i.e., the exact reproduction of an entire portion of data by oversampling the same pattern inside the TI. For these reasons, we recommend keeping the proposed standard value $T=0.05$ for all the variables.

- $F$ should be set sufficiently high to have a consistent choice of patterns but a value close to 1 - i.e., all of the TI is scanned each time - may lower the variability of the simulations and increase the verbatim copy. Using a training data set representative enough, the optimal value corresponds to a TI fraction containing some repetitions of the lowest-frequency fluctuation that should be reproduced. Considering the randomness of the TI scan, the value $F=0.5$ chosen in this paper is sufficient to serve the purpose.

\subsection{Imposing a trend}

As already shown in Chugunova and Hu (2008), Mariethoz et al. (2010), Honarkhah and Caers (2010) and $\mathrm{Hu}$ et al. (2014), in case of a nonstationary target variable, the simulation can be constrained to reproduce the same type of trend found in the TI by making use of an auxiliary variable. The one proposed here is the integer vector $L=[1,2, \ldots, M]$, where $M$ is the length of the time series, tracking the position of each datum inside the TI. $L$ is assigned to the SG as conditioning datum with the following parameters: $R_{L}=1$, $N_{L}=1$ and $T_{L}=0.01$. According to the threshold $T_{L}$, the sampling is therefore constrained to a neighborhood of the same time step inside the TI; for example, in the Darwin case, being $M=26356$ and $T_{L}=0.01$ ( $1 \%$ of the total variation allowed), the sampling to simulate $Z\left(x_{t}\right)$ is constrained to the interval $y_{t} \pm 263$ (days). In this way, the marginal distribution is respected, but the local variability is restricted to the one found inside the training data set, reproducing the 
same trend. The following remarks are noteworthy: (i) to avoid an unnecessary restriction of the sampling, $T_{L}$ should correspond to the maximum time interval for which the target variable can be considered stationary; (ii) the simulation should not be longer than the training data set, having no basis to extrapolate the trend in the past or future; (iii) the local variability is not completely limited by $L$ : a pattern outside the tolerance range (i.e., with a distance over the threshold) could be sampled if no better candidate is found.

\subsection{Validation}

To test the proposed technique, the visual comparison of the generated time series with the reference as well as several groups of statistical indicators is considered. The empirical cumulative probability distributions, obtained using the Kaplan-Meier estimate (Kaplan and Meier, 1958), of the daily, the annual and decennial rainfall time series, obtained by summing up the daily rainfall, are compared using quantile-quantile (qq) plots. Moreover, the minimum moving average - i.e., the minimum value found on the moving average of each time series - is computed using different running window lengths of up to 60 years to assess the efficiency of the algorithm in preserving the long-term dependence characteristics of the rainfall.

The daily rainfall statistics have been analyzed separately for each month considering the average value of the following indicators: the probability of occurrence of a wet day and the mean, standard deviation, minimum and maximum on wet days only. For instance, the standard deviation is computed on the wet days of each month of January, then the average value is taken as representative of that time series. We therefore obtain a unique value for the reference and a distribution of values for the simulations represented with a box plot.

Another validation criterion used is the comparison of the dry- and wet-spell-length distributions. Each series is transformed into a binary sequence with zeros corresponding to dry days and ones to the wet days. Then, counting the number of days inside each dry and wet spell, we obtain the distributions of dry- and wet-spell lengths, which can be compared using qq plots. This is an important indicator since it determines, for example, the efficiency of the algorithm in reproducing long droughts or wet periods.

Since DS works by pasting values from the TI to the SG, it is straightforward to keep track of the original location of each value in the training image. If successive values in the TI are also next to each other in the SG, then a patch is identified. A multiple box-plot is then used to represent the number of patches found in each realization as a function of the patch length to keep track of the verbatim-copy effect.

The last group of indicators considered is the sample partial autocorrelation function (PACF) (Box and Jenkins, 1976) of the daily, monthly and annual rainfall. Given a timeseries $\boldsymbol{X}_{t}$, the sample PACF is the estimation of the linear correlation index between the datum at time $t$ and those at previous time steps $t-h$, without considering the linear dependence with the in-between observations. For a stationary time series the sample PACF is expressed as a function of the time lag $h$ with the following formula:

$$
\begin{aligned}
\hat{\rho}\left(X_{t}, h\right)= & \operatorname{Corr}\left[X_{t}-\hat{E}\left(X_{t} \mid\left\{X_{t-1}, \ldots, X_{t-h+1}\right\}\right),\right. \\
& \left.X_{t-h}-\hat{E}\left(X_{t-h} \mid\left\{X_{t-h+1}, \ldots, X_{t-1}\right\}\right)\right],
\end{aligned}
$$

where $\hat{E}\left(X_{t} \mid\left\{X_{t-1}, \ldots, X_{t-h+1}\right\}\right)$ is the best linear predictor knowing the observations $\left\{X_{t-1}, \ldots, X_{t-h+1}\right\} . \rho(h)$ varies in the range $[0,1]$, with high values for a highly autocorrelated process. This indicator is widely used in time series analysis since it gives information about the persistence of the signal. The autocorrelation function could be used instead, but PACF is preferred here since it shows the autocorrelation at each lag independently. In the case of daily rainfall, the partial autocorrelation is usually very low, while the higher-scale rainfall may present a more important specific time-lag linear dependence. As usually done in the absence of any prior knowledge about $X_{t}$, the 5-95\% confidence limits of an uncorrelated white noise are adopted to assess the significance of the PACF indexes. Since the time series used in this paper are not necessarily stationary, any sample PACF is computed from the standardized signal $X_{t}^{\mathrm{s}}$, obtained by applying moving average estimation $\hat{m}_{t}$ and standard deviation $\hat{s}_{t}$ filters with the following formula:

$$
\begin{gathered}
X_{t}^{\mathrm{s}}=\frac{X_{t}-\hat{m}_{t}}{\hat{s}_{t}}, \hat{m}_{t}=(2 q+1)^{-1} \sum_{j=-q}^{q} X_{t+j}, \\
\hat{s}_{t}=\left[(2 q+1)^{-1} \sum_{j=-q}^{q}\left(X_{t+j}-\hat{m}_{t}\right)^{2}\right]^{-\frac{1}{2}}, \\
q+1 \leq t \leq n-q,
\end{gathered}
$$

where $q=2555$ (15-year centered moving window). It is important to note that this operation may exclude from the PACF computation a consistent part of the signal $(q+1 \leq t \leq n-q)$, especially on the higher timescale. In the case of the data sets used, the annual time series is reduced to less than 60 values for Alice Springs and Darwin: a barely sufficient quantity, considering that the minimum amount of data for a useful sample PACF estimation suggested by Box and Jenkins (1976) is of about 50 observations.

\section{Results and discussion}

To evaluate the proposed technique, a group of 100 realizations of the same length as the reference is generated for each of the three considered data sets to obtain a sufficiently stable response in both the average and the extreme behavior. The setup used is the one presented in Sect. 3 with the fixed parameter values shown in Table 2 . The obtained results are shown and discussed in the following section. 

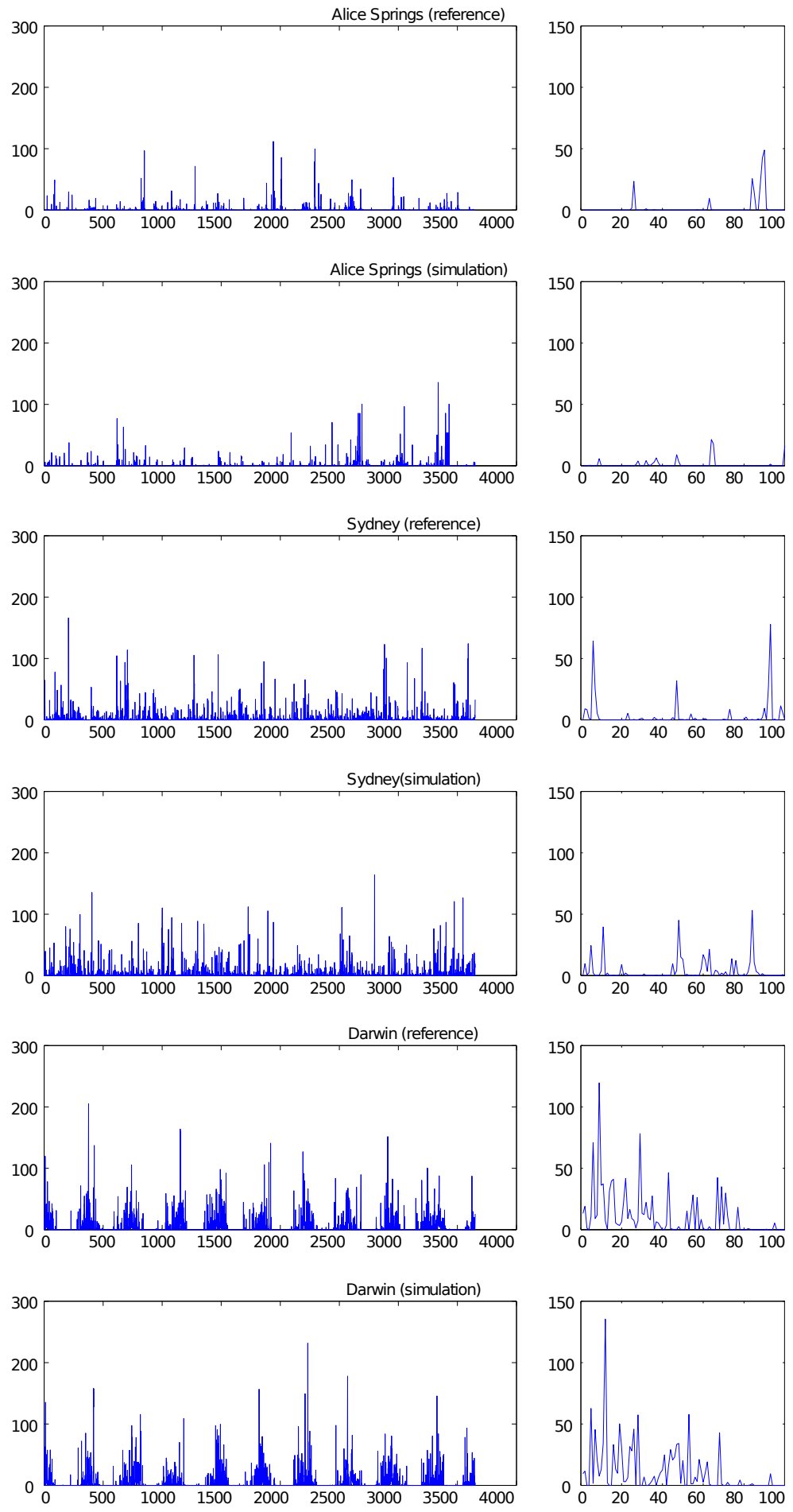

Figure 2. Visual comparison between the simulated and the reference daily rainfall $(\mathrm{mm})$ time series: 10-year (left-column panels) and 100-day (right-column panels) random samples.

\subsection{Visual comparison}

Figure 2 shows the comparison between random samples from both the simulated and the reference time series. For each data set, the generated rainfall looks similar to the reference: the extreme events inside the 10-year samples are reproduced with an analogous frequency and magnitude. The annual seasonality, particularly pronounced in the Darwin series, is accurately simulated as well as the persistence of the 
Alice Springs
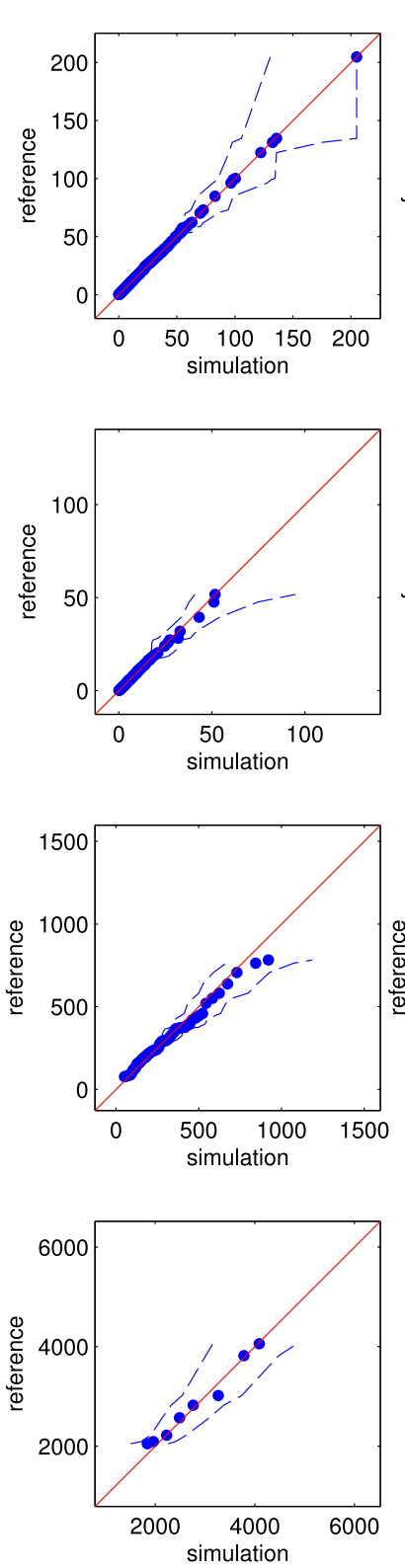

Sydney

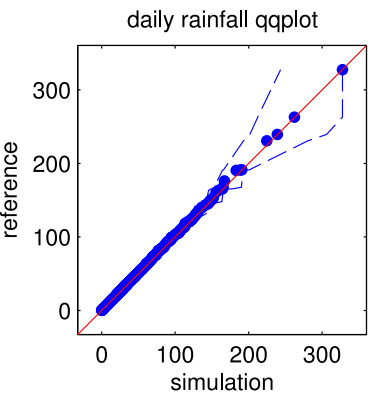

solitary wet days rainfall qqplot

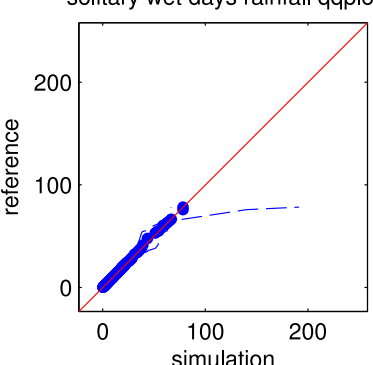

annual rainfall qqplot
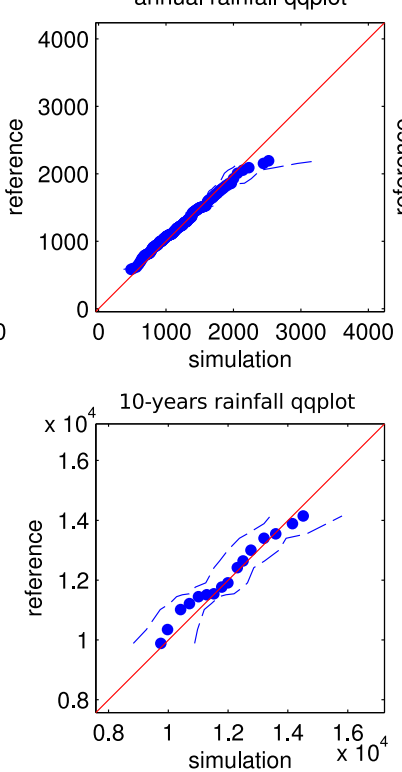

Darwin
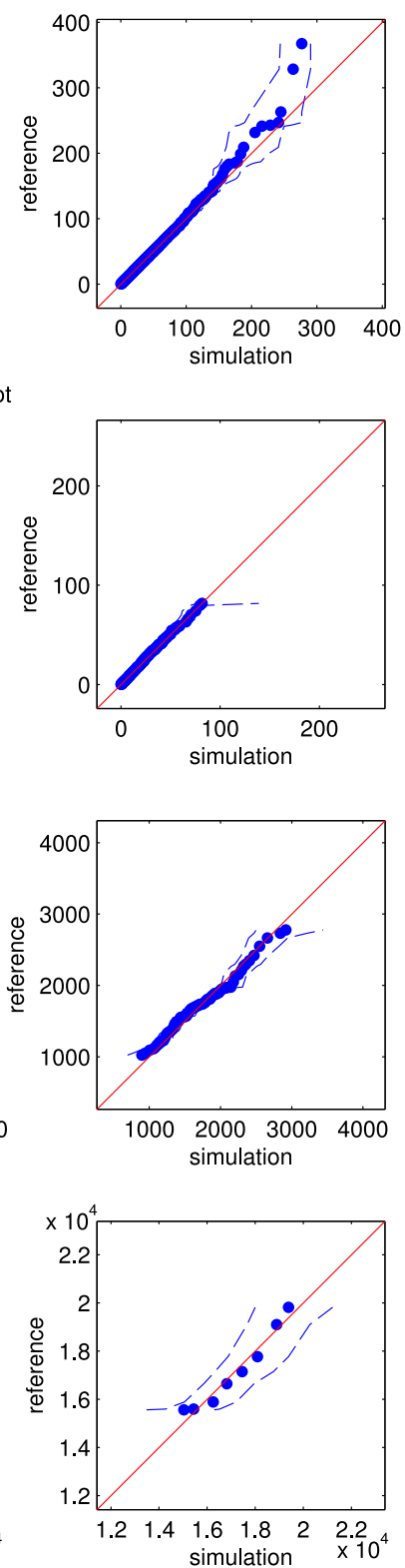

Figure 3. qq plots of the empirical probability rainfall amount $(\mathrm{mm})$ distributions: median of the realizations (blue dots), 5th and 95th percentiles (dashed lines). The bisector (solid line) indicates the exact quantile match.

rainfall events, visible in the 100-day samples. These aspects are evaluated quantitatively in the following sections.

\subsection{Multiple-scale probability distribution}

The qq plots of the rainfall empirical distributions are presented in Fig. 3, where all the range of quantiles is considered. The distribution of the daily rainfall (computed on wet days only) is generally respected, although some extremes that are present only once in the reference and, in particular, at the start or end of the time series, may not appear in the simulation. It is the case of the Darwin series, with a mismatch of the very upper quantiles. Moreover, being that the DS is an algorithm based on resampling, the distribution of the simulated values is limited by the range of the training data set: this is shown in the Alice Springs and Sydney qq plots, where the distribution of the last quantiles is clearly truncated at the maximum value found in the reference. This result is normally expected using this type of technique: the DS algorithm is of course not able to extrapolate extreme 

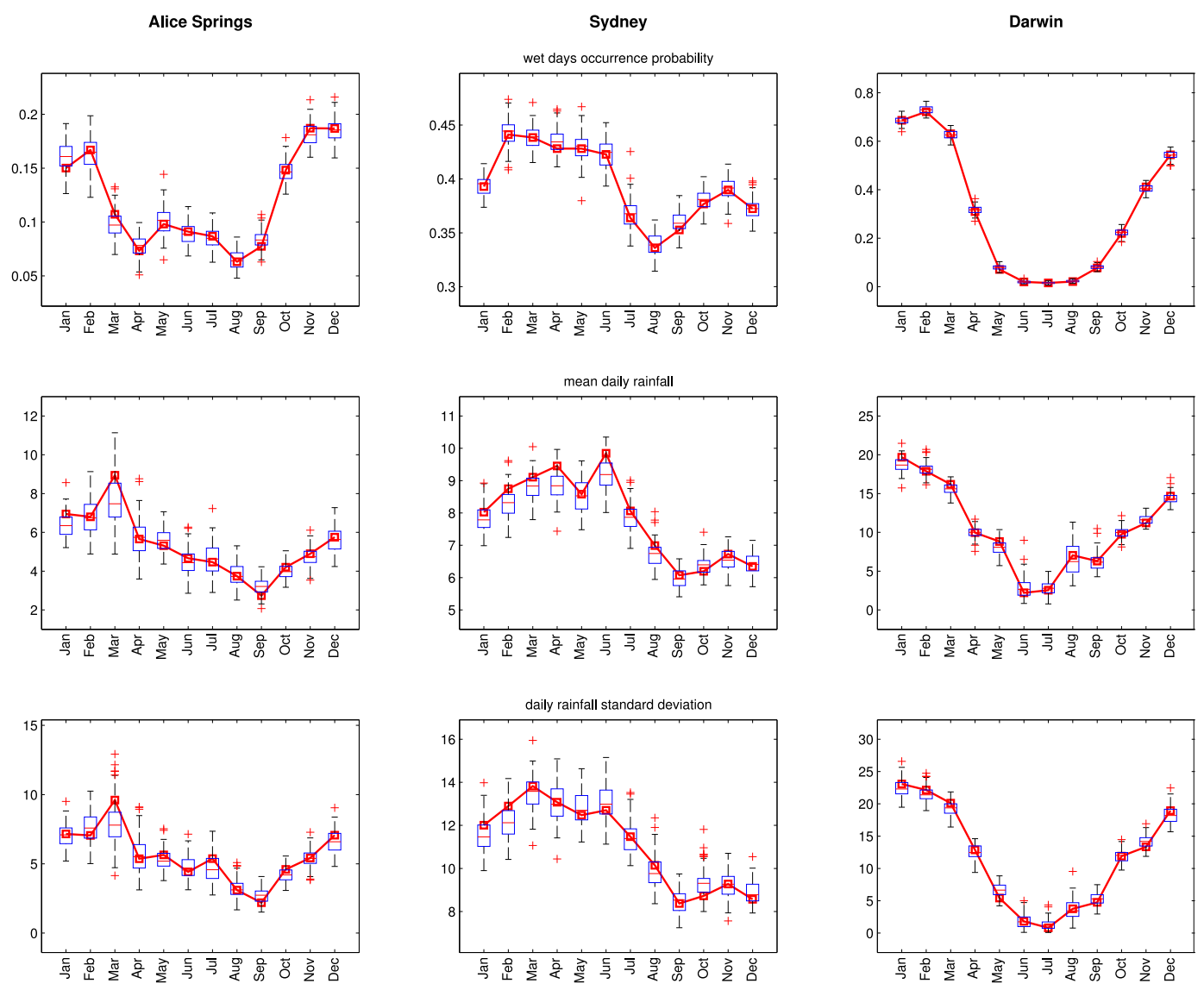

Figure 4. Box plots of the average wet-day probability, mean daily rainfall amount $(\mathrm{mm})$ and its standard deviation per month. The solid line indicates the reference.

intensities higher than the ones found in the TI at the scale of the simulated signal.

On the contrary, the distribution of the rainfall amount on the solitary wet days is accurately respected, with some realizations including higher extremes than the reference. More importantly, the annual and 10-year rainfall distributions are correctly reproduced and do not show overdispersion. This phenomenon, common among the classical techniques based on daily scale conditioning, consists in the scarce representation of the extremes and underestimation of the variance at the higher scale. This problem is avoided here because a variable dependence is considered, up to a 5000-day radius on the $365 \mathrm{MA}$ auxiliary variable, that helps preserving the low-frequency fluctuations. We also see that, at this scale, DS is capable of generating extremes higher than those found in the reference, meaning that new patterns have been generated using the same values at the daily scale. This results is purely based on the reproduction of higher-scale patterns: the acceptance threshold value chosen for the $365 \mathrm{MA}$ auxiliary variable allows enough freedom to generate new patterns although maintaining an unbiased distribution. Nevertheless, this approach is not meant to replace a specific technique to predict long recurrence-time events at any temporal scale, since it is not focused on modeling the tail of the probability distribution.

\subsection{Annual seasonality and extremes}

Figure 4 shows the principal indicators describing the annual seasonality of the reference and the generated time series: each different season is accurately reproduced by the algorithm, with almost no bias. The probability of having a wet day, usually imposed by a prior model in the classical parametric techniques, is indirectly obtained by sampling from the rainfall patterns of the appropriate period of the year. This goal is mainly achieved using the auxiliary variables tr 1 and tr2 as conditioning data (see Sect. 3).

The simulation of the average extremes, shown in Fig. 5, also follows the reference rather accurately.

\subsection{Rainfall patterns and verbatim copy}

The statistical indicators regarding the dry/wet patterns shown in Fig. 6 demonstrate the efficiency of the proposed DS setup in simulating long droughts or wet periods according to the training data set: the dry- and wet-spell 

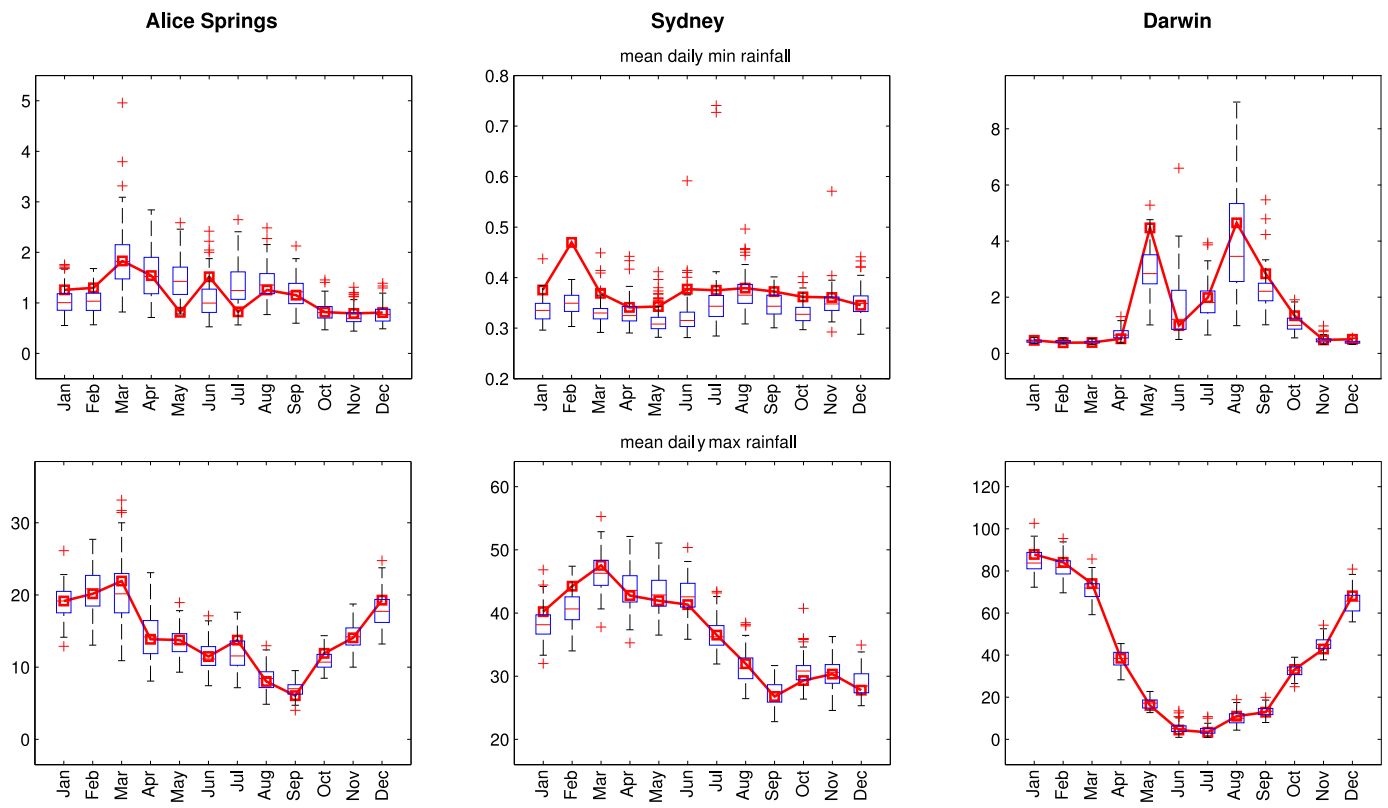

Figure 5. Box plots of the average extremes per month $(\mathrm{mm})$. The solid line indicates the reference.

distributions are preserved and extremes higher than the ones present in the TI are also simulated.

The verbatim-copy box plots show the distribution of the time series pieces exactly copied from the TI as a function of their size for the ensemble of the realizations: the number of patches decreases exponentially with their size. The phenomenon is mainly limited to a maximum of a few 8-day patches, with isolated cases of up to 14 days.

The 10-year rainfall moving sum, shown at the bottom of Fig. 6, illustrates the low-frequency time series structure: the quantiles of the simulations at each time step confirm that the overall variability is correctly simulated, but the local fluctuations do not match the reference. For example, the Darwin reference series shows a clear upward trend which is not present in the superposed randomly picked DS realization. Generally, the TI is supposed to be stationary or the nonstationarity should be at least described by an auxiliary variable. If it is not the case, as for the Darwin time series, the algorithm honors the marginal distribution of the reference, but it does not reproduce a specific trend. This problem is treated separately in Sect. 4.6.

The minimum moving average on different window lengths of up to 60 years (Fig. 7) gives information about the long-term structure of rainfall. The zero values are in accordance with the dry spell distribution shown in Fig. 6; for example, Alice Springs presents a zero-minimum moving average until 5 months, meaning that it contains dry spells of this length. Alice Springs and Sydney show a very different long-term structure: the former with long dry spells, the latter with a wider range of minimum values. Darwin presents the peculiarities of both climates with a sharp rising from the annual to the 60-year scale.

According to this indicator, the simulation of the longterm structure is fairly accurate. The negative bias, lower than $0.5 \mathrm{~mm}$, shows a modest tendency to underestimate the minimum moving average from the annual to the decennial scale for wet climates such as Sydney and Darwin.

\subsection{Linear time-dependence}

The specific linear time-dependence of the generated and reference signals has been evaluated at different scales using the sample PACF (Fig. 8, Eq. 4).

At the daily scale, the data show the same level of autocorrelation at lag 1 and a low but significant linear dependence until lag 3 for Alice Springs and Sydney, while Darwin presents a longer tailing which asymptotically approaches the confidence bounds of an uncorrelated noise. The DS simulation shows a tendency to a slight underestimation of the lag 1 PACF, with a maximum error around 0.1 for Sydney. Since the algorithm operates in a nonparametric way and imposes a variable time-dependence, the eventuality of modifying the structure of the daily signal cannot be excluded a priori, for this reason the PACF has been calculated up to the 20th lag, assuring that no extra linear-dependence has been introduced.

At the monthly scale, the linear time-dependence structure is clearly related to the annual seasonality, with a negative autocorrelation around lag 6 and a positive one around lag 12. The climate characterization is also evident: from Alice Springs to Darwin we see a more marked seasonality 

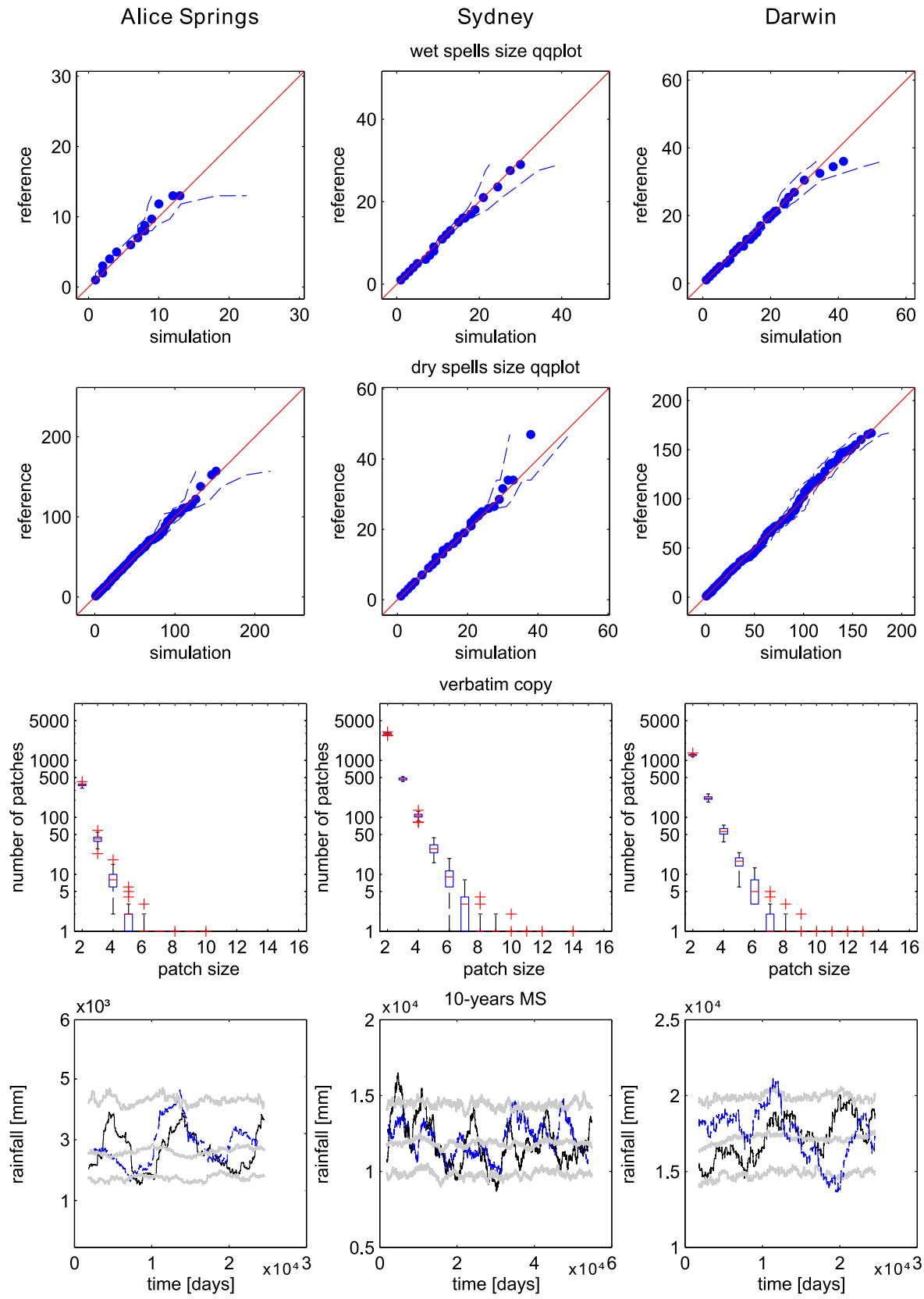

Figure 6. Main indicators describing the rainfall pattern: qq plots of the dry- and wet-spell (days) distributions, verbatim-copy box plots as function of the patch size (days) and daily 10-year MS time series (mm) of the reference (black line), median, 5th and 95th percentiles of the realizations (gray lines) and a randomly picked simulation (dashed blue line).

reflected in the PACF. The simulation follows the reference fairly well, with a maximum error of approximately \pm 0.1 .

At the annual scale, the limited length of the time series leads to wider confidence bounds for the nonsignificant values (see Sect. 3.2). The reference does not show a clear linear time-dependence structure which is not similarly reproduced by the simulation. Some more relevant discrepancies are present in the Darwin series, showing a more discontinuous structure. However, using such a limited data set for the timescale considered here, it is difficult to determine if the reference PACF is really indicative of an effective linear dependence.

\subsection{Nonstationary simulation}

Figure 9 shows the Darwin time-series simulation preserving the same nonstationarity contained in the reference by using the technique proposed in Sect. 3.1. The 10-year moving 

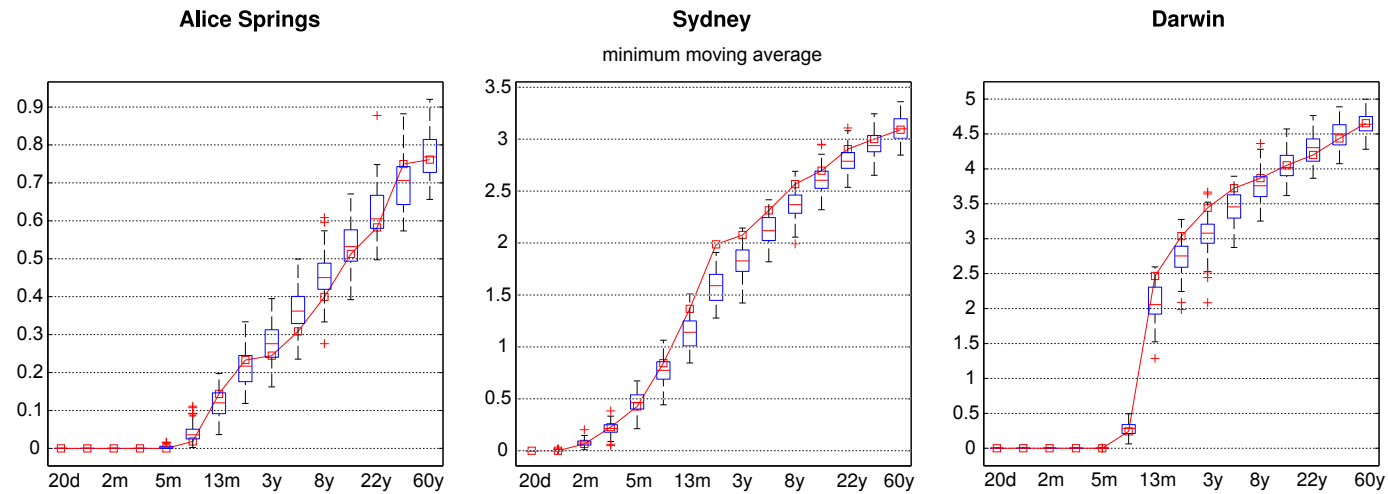

Figure 7. Minimum moving average of daily rainfall $(\mathrm{mm})$ for different running window lengths (days, months or years). The solid line indicates the reference.

Alice Springs
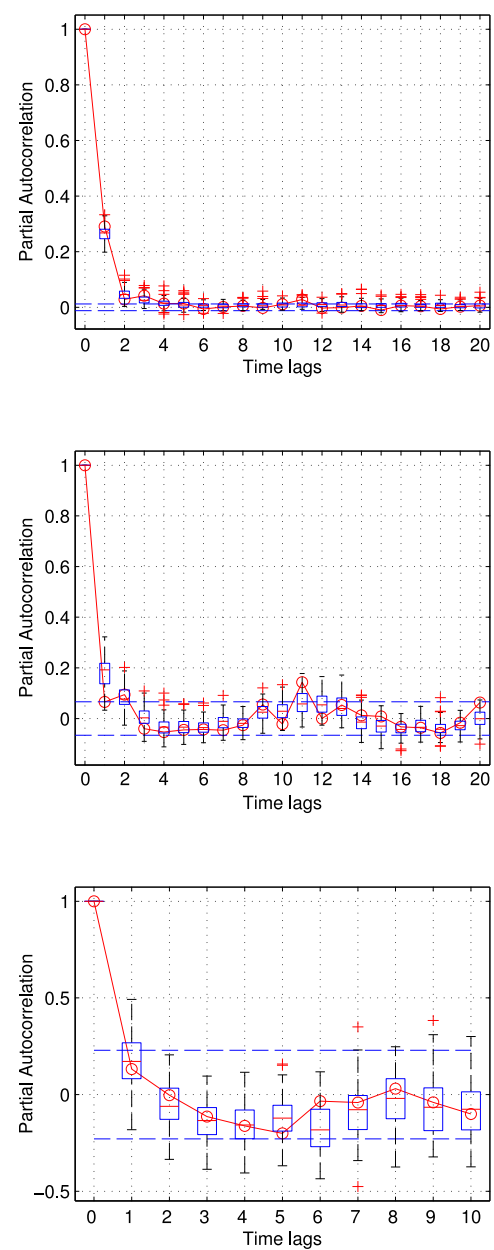

Sydney
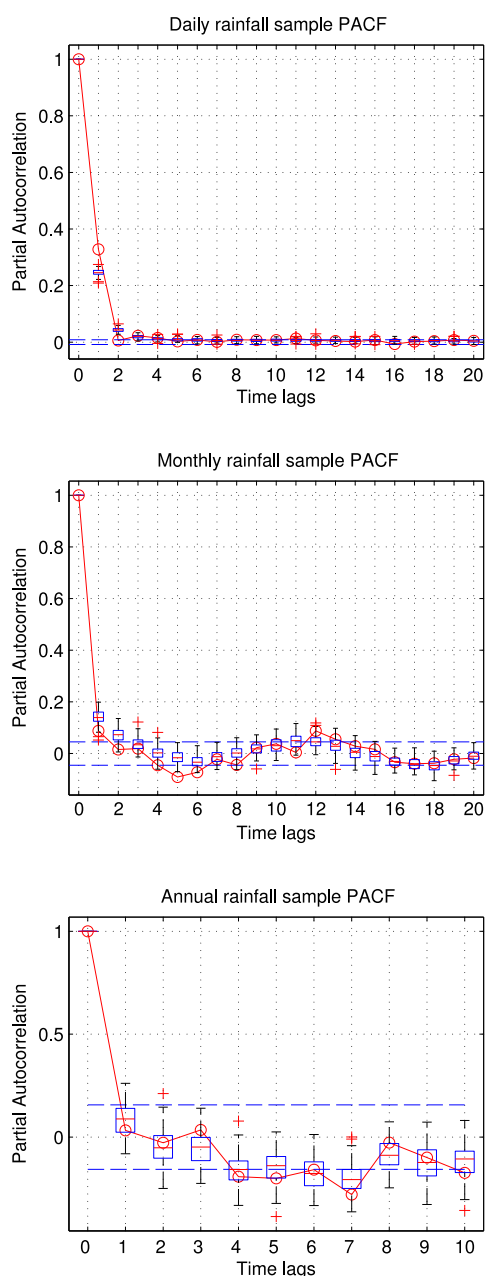

Darwin
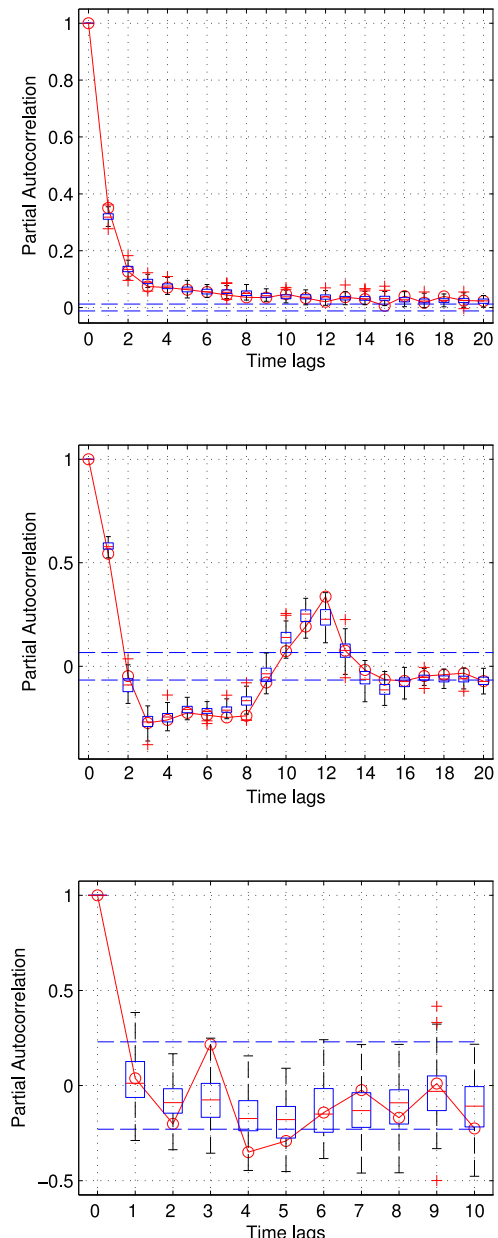

Figure 8. Sample PACF of the daily, monthly and annual rainfall signal. Reference (solid line), 100 DS simulations (box plots), and confidence bounds for the negligible autocorrelation indexes (dashed lines). 

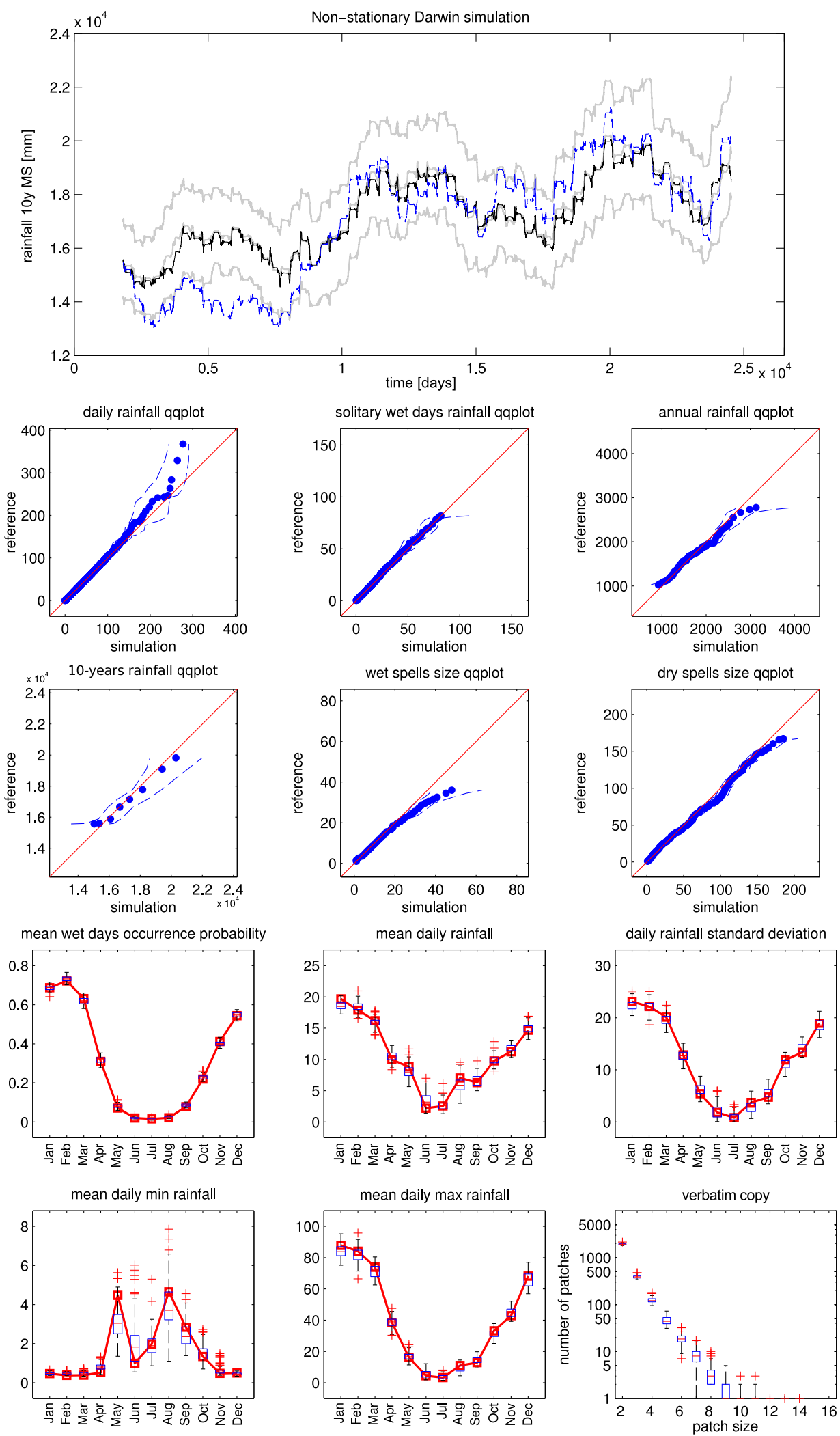

Figure 9. Darwin daily rainfall nonstationary simulation: 10-year moving sum time series (top panel) of the reference (black line), median, 5th and 95th percentiles of the realizations (gray lines) and a randomly picked simulation (dashed blue line); main quantile comparisons (center panels); main seasonal indicators and verbatim-copy box plots (bottom panels). 
sum plot shows that the trend and low-frequency fluctuation present in the reference are accurately simulated: the median of the realizations follows the reference and a variability of about $4 \mathrm{~m}$ between the 5 th and 95 th percentiles is present. Regarding the other considered statistical indicators, the performance appears to be essentially the same as for the stationary simulation: the only remarkable difference is a modest positive bias in the maximum wet-period length.

The fact that, to impose the trend, the sampling is restricted to a local region of the reference reduces the local variability with respect to the stationary simulation. Consequently, a modest increase of the verbatim-copy effect occurs.

This technique can be applied in cases where a specific nonstationarity extended to high-order moments should be imposed; e.g., exploring the uncertainty of a given past or future scenario, where a simple trend or seasonality adjustment is insufficient and an overly complex parametric model would be necessary to preserve the same long-term behavior.

\section{Conclusions}

The aim of the paper is to present an alternative daily rainfall simulation technique based on the direct sampling algorithm, belonging to the multiple-point statistics family. The main principle of the technique is to resample a given data set using a pattern-similarity rule. Using a random simulation path and a nonfixed pattern dimension, the technique allows imposing a variable time-dependence and reproducing the reference statistics at multiple scales. The proposed setup, suitable for any type of rainfall, includes the simulation of the daily rainfall time series together with a series of auxiliary variables including a categorical variable describing the dry/wet pattern, the 2-day moving sum which helps in respecting the lag 1 autocorrelation, the 365-day moving average to condition upon interannual fluctuations and two coupled theoretical periodic functions describing the annual seasonality. Since all the variables are automatically computed from the rainfall data, no additional information is needed.

The technique has been tested on three different climates of Australia: Alice Springs (desert), Sydney (temperate) and Darwin (tropical savannah). Without changing the simulation parameters, the algorithm correctly simulates both the rainfall occurrence structure and amount distribution up to the decennial scale for all the three climates, avoiding the problem of overdispersion, which often affects daily rainfall simulation techniques. Being based on resampling, the algorithm can only generate data which are present in the training data set, but they can be aggregated differently, simulating new extremes in the higher-scale rainfall and dry-/wetpattern distributions. The technique is not meant to be used as a tool to explore the uncertainty related to long recurrencetime events, but rather to generate extremely realistic replicates of the datum, to be used as inputs in hydrologic models.
Reproducing the specific trend found in the data is also possible by making use of an additional auxiliary variable which simply restricts the sampling to a local portion of the $\mathrm{TI}$. In this way, any type of nonstationarity present in the TI is automatically imposed on the simulation. The Darwin example demonstrates the efficiency of this approach in reproducing 100 different realizations showing the same type of trend and marginal distribution. This setup can be useful to simulate multiple realizations of a specific nonstationary scenario regardless of its complexity.

In conclusion, the direct sampling technique used with the proposed generic setup can produce realistic daily rainfall time-series replicates from different climates without the need of calibration or additional information. The generality and the total automation of the technique makes it a powerful tool for routine use in scientific and engineering applications.

Acknowledgements. This research was funded by the Swiss National Science Foundation (project no. 134614) and supported by the National Centre for Groundwater Research and Training (University of New South Wales). The data set used in this paper is courtesy of the Australian Bureau of Meteorology (BOM).

Edited by: E. Morin

\section{References}

Allard, D., Froidevaux, R., and Biver, P.: Conditional simulation of multi-type non stationary markov object models respecting specified proportions, Math. Geol., 38, 959-986, 2006.

Arpat, G. and Caers, J.: Conditional simulation with patterns, Math. Geol., 39, 177-203, 2007.

Bardossy, A. and Plate, E. J.: Space-time model for daily rainfall using atmospheric circulation patterns, Water Resour. Res., 28, 1247-1259, doi:10.1029/91WR02589, 1992.

Box, G. E. and Jenkins, G. M.: Time series analysis, forecasting and control, Holden-Day, Oakland, CA, 1976

Briggs, W. M. and Wilks, D. S.: Estimating monthly and seasonal distributions of temperature and precipitation using the new CPC long-range forecasts, J. Climate, 9, 818-826, doi:10.1175/1520 0442(1996)009<0818:EMASDO>2.0.CO;2, 1996.

Buishand, T. A. and Brandsma, T.: Multisite simulation of daily precipitation and temperature in the Rhine basin by nearestneighbor resampling, Water Resour. Res., 37, 2761-2776, doi:10.1029/2001WR000291, 2001.

Chugunova, T. L. and Hu, L. Y.: Multiple-point simulations constrained by continuous auxiliary data, Math. Geosci., 40, 133146, doi:10.1007/s11004-007-9142-4, 2008.

Clark, M. P., Gangopadhyay, S., Brandon, D., Werner, K., Hay, L., Rajagopalan, B., and Yates, D.: A resampling procedure for generating conditioned daily weather sequences, Water Resour. Res., 40, W04304, doi:10.1029/2003WR002747, 2004.

Coe, R. and Stern, R. D.: Fitting models to daily rainfall data, J. Appl. Meteorol., 21, 1024-1031, doi:10.1175/15200450(1982)021<1024:FMTDRD>2.0.CO;2, 1982.

Efron, B.: Bootstrap methods - another look at the jackknife, Ann. Statist., 7, 1-26, doi:10.1214/aos/1176344552, 1979. 
Guardiano, F. and Srivastava, R.: Multivariate geostatistics: beyond bivariate moments, Geostatistics-Troia, 1, 133-144, 1993.

Harrold, T. I., Sharma, A., and Sheather, S. J.: A nonparametric model for stochastic generation of daily rainfall occurrence, Water Resour. Res., 39, 1300, doi:10.1029/2003WR002182, $2003 \mathrm{a}$.

Harrold, T. I., Sharma, A., and Sheather, S. J.: A nonparametric model for stochastic generation of daily rainfall amounts, Water Resour. Res., 39, 1343, doi:10.1029/2003WR002570, 2003b.

Hay, L. E., Mccabe, G. J., Wolock, D. M., and Ayers, M. A.: Simulation of precipitation by weather type analysis, Water Resour. Res., 27, 493-501, doi:10.1029/90WR02650, 1991.

Honarkhah, M. and Caers, J.: Stochastic simulation of patterns using distance-based pattern modeling, Math. Geosci., 42, 487517, 2010

Hu, L. Y., Liu, Y., Scheepens, C., Shultz, A. W., and Thompson, R. D.: Multiple-Point Simulation with an Existing Reservoir Model as Training Image, Math. Geosci., 46, 227-240, doi:10.1007/s11004-013-9488-8, 2014.

Hughes, J. and Guttorp, P.: A class of stochastic models for relating synoptic atmospheric patterns to regional hydrologic phenomena, Water Resour. Res., 30, 1535-1546, 1994.

Hughes, J., Guttorp, P., and Charles, S.: A non-homogeneous hidden Markov model for precipitation occurrence, J. Roy. Stat. Soc. Ser. C, 48, 15-30, 1999.

Jones, P. G. and Thornton, P. K.: Spatial and temporal variability of rainfall related to a third-order Markov model, Agr. Forest Meteorol., 86, 127-138, doi:10.1016/S0168-1923(96)02399-4, 1997.

Kaplan, E. and Meier, P.: Non-parametric estimation from incomplete observations, J. Am. Stat. Assoc., 53, 457-481, doi:10.2307/2281868, 1958.

Katz, R. W. and Parlange, M. B.: Effects of an index of atmospheric circulation on stochastic properties of precipitation, Water Resour. Res., 29, 2335-2344, doi:10.1029/93WR00569, 1993.

Katz, R. W. and Zheng, X. G.: Mixture model for overdispersion of precipitation, J. Climate, 12, 2528-2537, doi:10.1175/15200442(1999)012<2528:MMFOOP>2.0.CO;2, 1999.

Kiely, G., Albertson, J. D., Parlange, M. B., and Katz, R. W.: Conditioning stochastic properties of daily precipitation on indices of atmospheric circulation, Meteorol. Appl., 5, 75-87, doi:10.1017/S1350482798000656, 1998.

Lall, U. and Sharma, A.: A nearest neighbor bootstrap for resampling hydrologic time series, Water Resour. Res., 32, 679-693, doi:10.1029/95WR02966, 1996.

Lall, U., Rajagopalan, B., and Tarboton, D. G.: A nonparametric wet/dry spell model for resampling daily precipitation, Water Resources Research, 32, 2803-2823, doi:10.1029/96WR00565, 1996.

Mariethoz, G. and Renard, P.: Reconstruction of Incomplete Data Sets or Images Using Direct Sampling, Math. Geosci., 42, 245268, doi:10.1007/s11004-010-9270-0, 2010.

Mariethoz, G., Renard, P., and Straubhaar, J.: The direct sampling method to perform multiple-point geostatistical simulations, Water Resour. Res., 46, W11536, doi:10.1029/2008WR007621, 2010.

Meerschman, E., Pirot, G., Mariethoz, G., Straubhaar, J., Van Meirvenne, M., and Renard, P.: A practical guide to performing multiple-point statistical simulations with the Direct Sampling algorithm, Comput. Geosci., 52, 307-324, doi:10.1016/j.cageo.2012.09.019, 2013.
Mehrotra, R. and Sharma, A.: A semi-parametric model for stochastic generation of multi-site daily rainfall exhibiting low-frequency variability, J. Hydrol., 335, 180-193, doi:10.1016/j.jhydrol.2006.11.011, 2007a.

Mehrotra, R. and Sharma, A.: Preserving low-frequency variability in generated daily rainfall sequences, J. Hydrol., 345, 102-120, doi:10.1016/j.jhydrol.2007.08.003, 2007b.

Ndiritu, J.: A variable-length block bootstrap method for multi-site synthetic streamflow generation, Hydrolog. Sci. J., 56, 362-379, doi:10.1080/02626667.2011.562471, 2011.

Rajagopalan, B. and Lall, U.: A k-nearest-neighhor simulator for daily precipitation and other weather variables, Water Resour. Res., 35, 3089-3101, doi:10.1029/1999WR900028, 1999.

Sharma, A. and Mehrotra, R.: Rainfall generation, in: Rainfall: state of the science, no. 191 in Geophysical Monograph Series, AGU, Washington, D.C., 215-246, 2010.

Srikanthan, R.: Stochastic generation of daily rainfall data using a nested model, 57th Canadian Water Resources Association Annual Congress, 16-18 June 2004, Montreal, Canada, 16-18, 2004.

Srikanthan, R.: Stochastic generation of daily rainfall data using a nested transition probability matrix model, in: 29th Hydrology and Water Resources Symposium: Water Capital, 20-23 February 2005, Rydges Lakeside, Canberra, p. 26, 2005.

Srikanthan, R. and Pegram, G. G. S.: A nested multisite daily rainfall stochastic generation model, J. Hydrol., 371, 142-153, doi:10.1016/j.jhydrol.2009.03.025, 2009.

Srinivas, V. V. and Srinivasan, K.: Hybrid moving block bootstrap for stochastic simulation of multi-site multi-season streamflows, J. Hydrol., 302, 307-330, doi:10.1016/j.jhydrol.2004.07.011, 2005.

Straubhaar, J.: MPDS technical reference guide, Centre d'hydrogeologie et geothermie, University of Neuchâtel, Neuchâtel, 2011.

Straubhaar, J., Renard, P., Mariethoz, G., Froidevaux, R., and Besson, O.: An improved parallel multiple-point algorithm using a list approach, Math. Geosci., 43, 305-328, 2011.

Strebelle, S.: Conditional simulation of complex geological structures using multiple-point statistics, Math. Geol., 34, 1-21, 2002.

Tahmasebi, P., Hezarkhani, A., and Sahimi, M.: Multiple-point geostatistical modeling based on the cross-correlation functions, Comput. Geosci., 16, 779-797, 2012.

Vogel, R. M. and Shallcross, A. L.: The moving blocks bootstrap versus parametric time series models, Water Resour. Res., 32, 1875-1882, doi:10.1029/96WR00928, 1996.

Wallis, T. W. R. and Griffiths, J. F.: Simulated meteorological input for agricultural models, Agr. Forest Meteorol., 88, 241-258, doi:10.1016/S0168-1923(97)00035-X, 1997.

Wang, Q. J. and Nathan, R. J.: A daily and monthly mixed algorithm for stochastic generation of rainfall time series, 27th Hydrology and Water Resources Symposium: Water Challenge - Balancing the Risks, 20-23 May 2002, Melbourne, Australia, p. 698, 2002.

Wilby, R. L.: Modelling low-frequency rainfall events using airflow indices, weather patterns and frontal frequencies, J. Hydrol., 212, 380-392, doi:10.1016/S0022-1694(98)00218-2, 1998.

Wilks, D. S.: Conditioning stochastic daily precipitation models on total monthly precipitation, Water Resour. Res., 25, 1429-1439, doi:10.1029/WR025i006p01429, 1989. 
Wojcik, R. and Buishand, T. A.: Simulation of 6-hourly rainfall and temperature by two resampling schemes, J. Hydrol., 273, 69-80, doi:10.1016/S0022-1694(02)00355-4, 2003.

Wojcik, R., McLaughlin, D., Konings, A., and Entekhabi, D.: Conditioning stochastic rainfall replicates on remote sensing data, IEEE T. Geosci. Remote, 47, 2436-2449, 2009.

Woolhiser, D. A., Keefer, T. O., and Redmond, K. T.: Southern oscillation effects on daily precipitation in the southwestern United-States, Water Resour. Res., 29, 1287-1295, doi:10.1029/92WR02536, 1993.
Young, K. C.: A multivariate chain model for simulating climatic parameters from daily data, J. Appl. Meteorol., 33, 661-671, doi:10.1175/1520-0450(1994)033<0661:AMCMFS>2.0.CO;2, 1994.

Zhang, T., Switzer, P., and Journel, A.: Filter-based classification of training image patterns for spatial simulation, Math. Geol., 38, 63-80, 2006. 